\title{
Arqueoastronomía y Astronomía Cultural, sus campos de acción y aplicación en Honduras - Conceptos
}

Eduardo Enrique Rodas Quito

\section{Resumen}

Se investigaron las posibles diferencias que podrían existir entre las disciplinas que se conocen como Arqueoastronomía y Astronomía Cultural, con el propósito general de obtener un perfil del campo de aplicación de ambas disciplina que sea apropiado para su uso en Honduras. Al hacer una revisión bibliográfica de varias obras que tratan este tema, se encontraron diferentes definiciones que se han dado sobre estas disciplinas, las que se evaluaron con el propósito de contar con una aproximación a esta temática, desde un punto de vista conceptual y relacionarlo con la realidad hondureña. Como resultado de este trabajo, se propone designar la disciplina como Astronomía Cultural, dado que éste es un término amplio para designar el estudio de la relación entre las diferentes culturas (ya sean antiguas o contemporáneas) y los fenómenos y cuerpos celestes, lo que es ampliamente discutido y argumentado en el documento que sigue a continuación. Además, se propone una posible estrategia para encontrar la relación de cercanía de la Astronomía Cultural con las ciencias físicas y las humanísticas, lo que vendría a ser material para futuros trabajos de investigación en el tema.

Palabras claves: Astronomía Cultural, Arqueoastronomía, Arqueología, Antropología, Astronomía.

\section{Abstract}

The possible differences that may exist between the disciplines known as Archaeoastronomy and Cultural Astronomy are investigated, with the general purpose of obtaining a profile of the scope of both sciences that can be applicable to Honduras. Upon making a bibliographic revision of documents related to this matter, 
it was found that various definitions have been given for both disciplines, which were evaluated in order to have a wide perspective on this issue, from a conceptual point of view and related to the honduran situation. As a result, it is proposed that both disciplines are designated by the terms "Cultural Astronomy", since these are comprehensive of the study of the relation that different cultures (whether they are ancient of contemporary) and the celestial bodies and phenomena, as it is discussed and supported in the following document. Also, a possible strategy to find the proximity of the Astronomical Culture to the physical and Humanistic Sciences is proposed, which could be the starting point for future studies on this matter.

Keywords: Cultural Astronomy, Archaeoastronomy, Archaeology, Anthropology, Astronomy.

Eduardo Enrique Rodas Quito, Departamento de Arqueoastronomía y Astronomía Cultural, Universidad Nacional Autónoma de Honduras 


\section{Introducción}

La Arqueoastronomía y la Astronomía Cultural son dos disciplinas muy recientes, si recordamos que la primera tiene sus antecedentes en los trabajos pioneros de Sir Norman Lockyer, apenas hacia finales del siglo XIX, cuando publicó su obra "The Dawn of Astronomy", mientras la Astronomía Cultural es aún mas reciente, ya que no se le cita por primera vez sino hasta el año 1990 (Iwaniszewski, 1990) (Iwaniszewski, 1991)(Ruggles / Saunders, 1990). Ambas disciplinas tienen objetos de estudio muy similares: la astronomía y su relación con las diferentes culturas que han poblado el planeta Tierra. Esta similitud entre ellas así como el poco tiempo que ha transcurrido para que se haya dado una completa difusión de estas disciplinas han provocado que a la hora de diferenciarlas por gran parte de la comunidad científica así como del público en general exista confusión sobre el alcance y por tanto, de las diferencias entre cada una de ellas. Por estos motivos, en el presente estudio se revisan diversos conceptos de Astronomía Cultural y de Arqueoastronomía, comparándolos y extrayendo de ellos un concepto que sirva para empezar a diferenciarlas y que al mismo tiempo puedan servir como una guía que describa la actividad que se desarrolla en el departamento de Arqueoastronomía y Astronomía Cultural, perteneciente a la Facultad de Ciencias Espaciales (FACES) de la Universidad Nacional Autónoma de Honduras, orientando los trabajos futuros de dicho departamento en este campo del conocimiento humano. Asimismo, se exploran los posibles límites que existan entre los campos de acción entre la Arqueoastronomía, la Astronomía Cultural y otras ciencias relacionadas, las cuales tienen algunos aspectos o metodologías útiles para estudiar cómo las culturas (antiguas y modernas) fueron y son influenciadas por los fenómenos astronómicos.

\section{Metodología}

La metodología utilizada se basó en la investigación documental, es decir, la búsqueda de documentos tales como artículos de revistas especializadas, informes de investigaciones previas sobre el tema aplicados a otros países y libros de texto que incluyeran como parte de su contenido el tema del alcance y objeto de estudio de las disciplinas de la Arqueoastronomía y/o Astronomía Cultural. Una vez hecho lo anterior, se procedió al análisis de dichos documentos en busca de elementos tomados en cuenta por los autores para llevar a cabo sus estudios así como sus resultados. Asimismo, se analizaron las ciencias involucradas y las metodologías de análisis utilizadas, para de esta manera tener un panorama de hacia donde se han orientado las investigaciones llevadas a cabo hasta la fecha y pro- 
poner un esquema de trabajo apropiado para el caso de Honduras. A partir de esto, se localizaron y revisaron varios documentos que hablan del tema tal como se ha aplicado en otros países, entre los cuales destaca el caso de España, donde varios autores han escrito varias reflexiones sobre sus experiencias en dicho país (Cerdeño et al., 2006)(Cerdeño Serrano \& Rodríguez Caderot, 2009)(Esteban, 2009).

\section{Resultados y Discusión}

Como ya se mencionó, se revisaron los escritos de varios autores que han trabajado en las disciplinas de la Arqueoastronomía y Astronomía Cultural. Muchos de estos escritos se elaboraron entre 1995 y 2008, período en el que surge una autocrítica entre los arqueoastrónomos sobre el rumbo que debería tomar la disciplina de la Arqueoastronomía y sobre la importancia que estaba adquiriendo la Astronomía Cultural como una novedosa forma de estudio de las culturas y las influencias que tuvieron por parte de los fenómenos astronómicos. Los artículos mas recientes (año 2008 hasta el presente) parecen tener mas claro el debate, en cuanto a que se refieren a cuál debería ser el rol de la Arqueoastronomía respecto a otras ciencias, tales como la Arqueología, la Antropología y la Astronomía. Se ha encontrado que en varios de los documentos consultados y que corresponden a años recientes, sus autores coinciden en que la Arqueoastronomía es una parte de la Astronomía Cultural, teniendo ésta última un carácter mas general y globalizador que la primera (Aveni, 2008) (Iwaniszewski, 2009), ya que abarca tanto a la arqueoastronomía (cuando estudia culturas antiguas y/o desaparecidas) como a la etnoastronomía (que tiene que ver con la astronomía de los grupos étnicos contemporáneos o también llamadas culturas vivas), además de abordar las percepciones y actitudes humanas hacia los fenómenos celestes desde un punto de vista mas amplio. Algunos autores se han dado a la tarea de proponer definiciones para la disciplina comentada en sus artículos, de esta forma se encontró que para la arqueoastronomía, estas definiciones van entre dos extremos, desde la que las clasifica a ésta como una disciplina muy próxima a, si no es que al servicio de otras de tipo social como la arqueología e historia de religiones (García Quintela \& González García, 2009)(Belmonte Avilés, 2006-2007), hasta las que la definen como una de tipo multidisciplinar pero enfocada en la astronomía propiamente dicha (Esteban, 2003), pasando por las definiciones de tipo intermedio en las que se afirma que el estudio humanístico y el astronómico están muy entrelazados (Ruggles, 2005). Respecto a la Astronomía Cultural, en todas ellas se incorpora el término "cultura", lo cual no es de extrañar, pero que tiene implicaciones profundas como se discute más adelante. 
En las definiciones mencionadas anteriormente llama la atención que a la Arqueoastronomía se la coloca "al servicio de" otras ciencias, mientras que la Astronomía Cultural es considerada una disciplina autónoma, que incluso puede tener diversas formas de aproximarse a su objeto de estudio. Se puede inferir que la Astronomía Cultural es mucho mas amplia y abarcadora del tema que le interesa, no está supeditada a otras ciencias, a diferencia de la Arqueoastronomía, que sí lo está y está limitada temporalmente a las culturas antiguas y prehistóricas. De modo que al tener un carácter mas general, la Astronomía Cultural abarcaría a la Arqueoastronomía, de hecho, "la Astronomía Cultural puede ser el marco adecuado en el que incluir todos los estudios que contribuyen a conocer la percepción que las sociedades antiguas tenían del cielo y sus fenómenos" (Cerdeño Serrano \& Rodríguez Caderot, 2009). Esto concuerda con lo que escribe otro autor, Clive Ruggles, cuando dice que "los arqueoastrónomos están preparados para [estudiar] una amplia gama de evidencias - no solo arqueológicas - de modo que no solo se restringen al estudio de alineamientos de monumentos. En este sentido la palabra 'arqueoastronomía' lleva a confusión, sin embargo el término ha permanecido en uso" (Ruggles, 2005). En cuanto a la Astronomía Cultural, las definiciones son a nuestro criterio mas abarcadoras, en cuanto a que incluyen el concepto de cultura dentro de las mismas (Ruggles \& Saunders, The Study of Cultural Astronomy, 2008), el que ya es amplio de por sí, así como a que se refieren a varios aspectos mas relacionados con la actividad humana de la incorporación e interpretación de elementos naturales dentro de las sociedades (Iwaniszewski, 2009).

Desde un punto de vista operacional, la Arqueoastronomía y la Astronomía Cultural no son consideradas ni ciencias físicas ni tampoco ciencias humanísticas, sino un híbrido entre ambas. Uno de los autores propone que quien se dedica a ellas, debería ser un "astrónomo reciclado" o un antropólogo "reciclado", es decir, un profesional de cualquiera de esas dos carreras pero "olvidando muchas de las referencias epistemológicas aprendidas en sus largos años de formación y aprendiendo otras nuevas que le eran completamente desconocidas" (Belmonte Avilés, 2006-2007). A pesar que han sido los astrónomos quienes tradicionalmente han tomado la iniciativa en el estudio de esta ciencia, partiendo desde la astronomía, "esta perspectiva ... resulta incompleta, por ello el punto de vista de los arqueólogos no debería estar ausente, puesto que no se trata de solo observar cuerpos celestes, de descubrir orientaciones o de tomar medidas de monumentos antiguos, sino de aproximarnos a la visión que de todo ello tenían los grupos humanos que los construyeron" (Cerdeño Serrano \& Rodríguez Caderot, 2009). De este modo, mientras no exista una institución / organismo / departamento universitario que se dedique en forma exclusiva a ambas disciplinas (arqueoastronomía y astronomía 
cultural) éstas pueden tener cabida en escuelas orientadas ya sea a las ciencias astronómicas o a las ciencias de estudio del hombre, es decir, que escuelas universitarias que tengan un interés genuino en llevar cabo trabajos de investigación en Arqueoastronomía y/o Astronomía Cultural pueden dar cobijo y atribuirse los resultados obtenidos en las investigaciones hechas en estos campos de estudio. Lo importante es que de dichos trabajos se obtenga información que sea de utilidad para la mejor comprensión de la dinámica y los comportamientos de sociedades que son generalmente diferentes de la llamada Cultura Occidental (es decir, la que tiene su origen en la cultura de los países colonizadores de los siglos XVI, XVII, XVIII y XIX de nuestra era, que son los del occidente de Europa, entre ellos España, Reino Unido y Francia), utilizando para ello herramientas metodológicas que sean apropiadas para dichos estudios, es decir, las que incorporen conocimientos de la astronomía y la antropología. Esta metodología no se menciona en los artículos evaluados, por lo que se vuelve necesario revisar en trabajos arqueoastronómicos hechos hasta ahora cuales han sido las metodologías utilizadas así como la disciplina mas afín a las mismas. Entonces se podría tener una mejor idea acerca de la proximidad de la astronomía cultural con las ciencias físicas y las humanísticas.

En resumen, podríamos decir que luego de un período de mucho debate sobre la naturaleza de la Arqueoastronomía, período en el cual surge la Astronomía Cultural como una disciplina más amplia a nivel conceptual, se ha llegado a una época en la que está claro el rol de cada una de ellas, habiendo consenso en que la Arqueoastronomía es parte de la Astronomía Cultural, quedando claramente definido que la primera se refiere al estudio de culturas antiguas, que ya no existen, mientras la segunda abarca, además de las culturas antiguas, también a las culturas actuales, las que son objeto de estudio de otras disciplinas tales como la Etnoastronomía. Debido a esto es que al referirse al estudio de las culturas y su relación o influencia de los fenómenos astronómicos de una manera general, se propone el uso de los términos "Astronomía Cultural" en lugar de solo hablar de Arqueoastronomía o Etnoastronomía de forma aislada, de esta manera evitando crear ambigüedades al estudiar culturas de larga tradición y cuyos descendientes podemos todavía identificar y estudiar para encontrar en ellos rasgos culturales que pudieran dar pistas sobre tradiciones o prácticas que eran habituales en sus antepasados (lo que es mas común de lo que uno podría pensar, por ejemplo, el caso de los mayas), por tanto, existiendo la duda en la clasificación de un trabajo de este tipo: se refiere a arqueoastronomía o a etnoastronomía? No cabe duda que con Astronomía Cultural, dicha ambigüedad queda descartada. En el caso de Honduras, esto vendría a ser de gran ayuda, porque nuestro país cuenta con una diversidad de grupos étnicos con su propia cultura cada una de ellas, tal es el caso 
de los indios Pech en el norte de Olancho, los tolupanes al norte del departamento de Francisco Morazán, o los lencas del occidente del país, por citar algunos ejemplos. En ellos existen rasgos culturales relacionados con la astronomía que vale la pena estudiar. ¿Estas tradiciones son propias de ellas o heredados de otras etnias? ¿Se pueden relacionar algunos restos arqueológicos con dichos grupos étnicos? Si es así, solo la Astronomía Cultural puede realizar un estudio integral tanto de la evidencia antigua como de la moderna.

Por otro lado, es recomendable evaluar las metodologías utilizadas hasta ahora en los estudios relacionados con la Astronomía Cultural a fin de conocer la proximidad de esta disciplina con las ciencias físico / matemáticas y las de tipo humanístico. También, es necesario implementar un sistema de manejo de base datos de la literatura existente en relación con religión, mitologías, cosmogonías de las culturas para un mejor acceso al mismo, ya que por la naturaleza de la disciplina de la Astronomía Cultural, se debe recurrir a literatura que tienen que ver tanto con ciencias humanísticas (como antropología o arqueología) como con ciencias físicas (astronomía observacional o mecánica celeste), las que no están unificadas en una sola base de datos, sino que hay que localizarla en fuentes muy dispares, según la naturaleza de la información que se está buscando.

\section{Bibliografía}

- Aveni, A., (2008) Introduction, Foundations of New World Cultural Astronomy, 2.

- Belmonte Aviles, J. A., (2006-2007) De la Arqueoastronomía a la Astronomía Cultural, Boletín de la SEAC, 15.

- Cerdeño Serrano, M. L., Rodríguez-Caderot, G. (2009) Arqueoastronomía: Una nueva perspectiva en la investigación arqueológica, Complutum, 20, 2, 11 - 21

- Cerdeño Serrano, M.L., Rodríguez-Caderot, G., Moya, P., Ibarra, A., Herrero, S. (2006) Los Estudios de Arqueoastronomía en España: Estado de la Cuestión, Trabajos de Prehistoria, 63, No.2, 13-34

- Esteban, C. (2009) La Astronomía Cultural es interdisciplinar? Reflexiones de un Astrofísico, Complutum,20, 2, 69-77.

- García Quintela, M., González García, C. (2009) Arqueoastronomía, Antropología y Paisaje, Complutum, 20,2, 39-54. 
- Iwaniszewski, S. (1990) Astromlmiia kak kul'lurnaia sislema. Na mht':.hakh po:nun;ia I,,\{t'Il'Il' noy, Nauka, Moskva, 67 - 73

- Iwaniszewski, S. (1991) Astronomy as a cultural system, Interdisciplinarni izsledvaniya, 18, pp. 282 - 288

- Iwaniszewski, S. (2009) Por una Astronomía Cultural Renovada. Complutum, 20(2), pp. $23-37$

- Ruggles, C. (2005) Ancient Astronomy - An Encyclopedia of Cosmologies and Myth, 19-20, 52, 169.

- Ruggles, C. Saunders, N (1990) The Study of Cultural Astronomy (Ponencia en la Conferencia Internacional sobre Arqueoastronomía "Oxford 3": Astronomers and Cultures), 